\title{
IMPROVEMENT OF CROSS-CORRELATION TECHNIQUE FOR LEAK DETECTION OF A BURIED PIPE IN A TONAL NOISY ENVIRONMENT
}

\author{
DOO-BYUNG YOON*, JIN-HO PARK, and SUNG-HWAN SHIN \\ Nuclear Technology Convergence Division, KAERI \\ 1045 Daedeok-daero, Yuseong-gu, Daejeon, 305-353, Korea \\ *Corresponding author. E-mail : yoondb@kaeri.re.kr
}

Received November 23, 2011

Accepted for Publication March 21, 2012

The cross-correlation technique has been widely used for leakage detection of buried pipes, and this technique can be successfully applied when the leakage signal has a high signal-to-noise ratio. In the case of a power plant, the measured leakage signals obtained from the sensors may contain background noise and mechanical noise generated by adjacent machinery. In such a case, the conventional method using the cross-correlation function may fail to estimate the leakage point. In order to enhance the leakage estimation capability of a buried pipe in a noisy environment, an improved cross-correlation technique is proposed. It uses a noise rejection technique in the frequency domain to effectively eliminate the tonal noise due to rotating machinery. Experiments were carried out to verify the validity of the proposed method. The results show that even in a tonal noisy environment, the proposed method can provide more reliable means for estimating the time delay of the leakage signals.

KEYWORDS : Leakage Detection, Buried Pipe, Cross-correlation, Noise Reduction, Signal Processing

\section{INTRODUCTION}

The importance of leak detection of buried pipes in a power plant of Korea is being emphasized as the buried pipes of the power plant are more than 20 years old. Various leak detection methods such as the pressure wave detection technique[1], the ultrasonic guided wave technique[2], the thermography method[3], and the process model-based method[4] have been proposed and implemented over the past years. Some of the most important are summarized in reference [5].

The cross-correlation method is one of the most commonly used methods for leak detection of buried pipes. This technique has been shown to be effective and is in common use in the water industry[6 10]. A leak from a buried pipe generates a leakage signal, which can be used for leak point detection. The leak position can be identified by the delay between the leakage signals propagating to each monitoring point as shown in Fig.1.

The cross-correlation techniques can be successfully applied when the leakage signal has a high signal-to-noiseratio(SNR). However, in case of a power plant, lots of machinery is installed and continuously operated without a break. The measured signals obtained from the vibration sensors may contain background noise and machinery noise components as well as a leakage signal component as shown in Fig.2. Since the effectiveness of the cross- correlation technique is largely influenced by the background noise $[8,10]$, in the case of a power plant, the crosscorrelation technique can be problematic for locating leaks. As one of method for enhancing SNR of the leakage signal, Kazuhisa[11] proposed a method for removing background noise by using a noise canceller and additional vibration sensors installed on the ground. The noise other than leakage signals is removed by noise cancellers comprised of adaptive digital filters by using the signal of a ground installed vibration sensor. However, this method requires additional sensors, data acquisition hardware, and more complex adaptive filters to cancel the background noise. This method may not be the best for leak detection of a power plant, where speedy leak inspection and rapid movement/reinstallation of the inspection equipment is necessary.

The objective of this work is to investigate the feasibility of the cross-correlation technique for leak detection of buried pipes in a power plant. For this purpose, the effect of the rotating machinery noise on the cross-correlation technique is investigated by performing numerical simulations. Then, to improve the leak detection capability of the cross-correlation technique in such a noisy environment, a modified cross-correlation method that can effectively remove the rotating machinery noise component is suggested. In addition, the validity of the proposed method is verified by performing an experiment. 


\section{IMPROVEMENT OF CROSS-CORRELATION TECHNIQUE}

In order to detect and locate a leak, the cross-correlation technique is commonly used to estimate the time delay between two vibration signals measured on either side of the leak. Since the cross-correlation technique can be successfully applied when the leakage signal has a high signal-to-noise ratio, the basic assumption of this work is that there is no flow in the pipe. Fig. 3 shows the schematic diagram of a typical measurement arrangement for leak detection of a buried pipe in a power plant. Vibration sensors can be attached on each side of a buried pipe. On the ground of the power plant, a number of machines are installed and continuously operated without a break. For the most part, the machinery involves a rotating mechanism such as a pump, motor, or cooling fan. The presence of the rotating machinery is highly undesirable from the perspective of leak detection, as it produces mechanical noise.

Assume that the leak signals are measured by two accelerometers in the presence of the background noise due to operation of the rotating machinery. This can be modeled as

$$
\begin{aligned}
& x_{1}(t)=s_{1}(t)+n_{1}(t) \\
& x_{2}(t)=s_{2}(t)+n_{2}(t)
\end{aligned}
$$

where $s_{1}(t)$ and $s_{2}(t)$ represent the leakage signals measured at sensor locations 1 and 2, respectively. $n_{1}(t)$ and $n_{2}(t)$ represent the machinery noise signals measured at sensor locations 1 and 2, respectively.

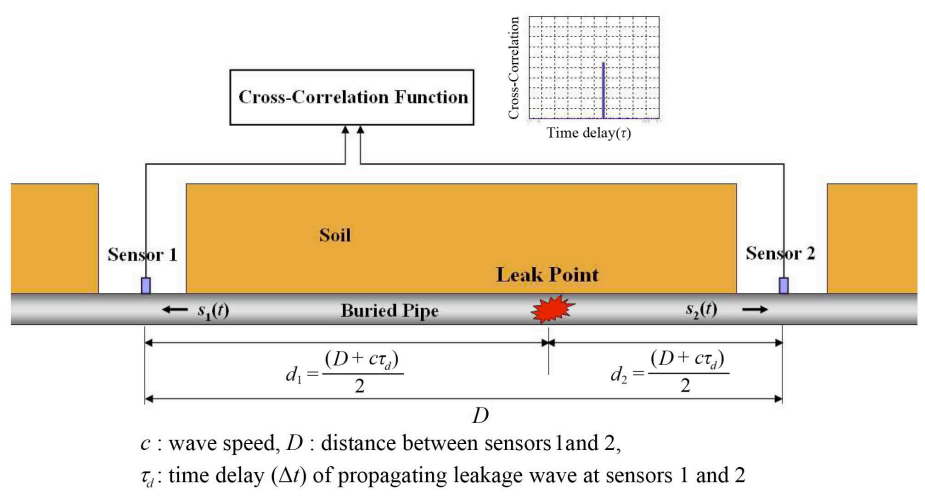

Fig. 1. Typical Measurement Arrangement of Cross-correlation Technique for Leak Detection of a Buried Pipe
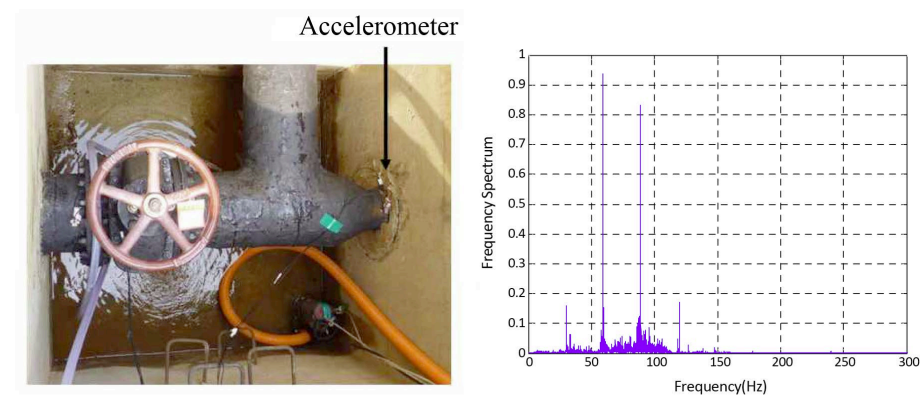

Fig. 2. Example of the Measured Vibration Signal Due to Leakage at the Valve of a Power Plant (Leak Rate: 3 liter/min)

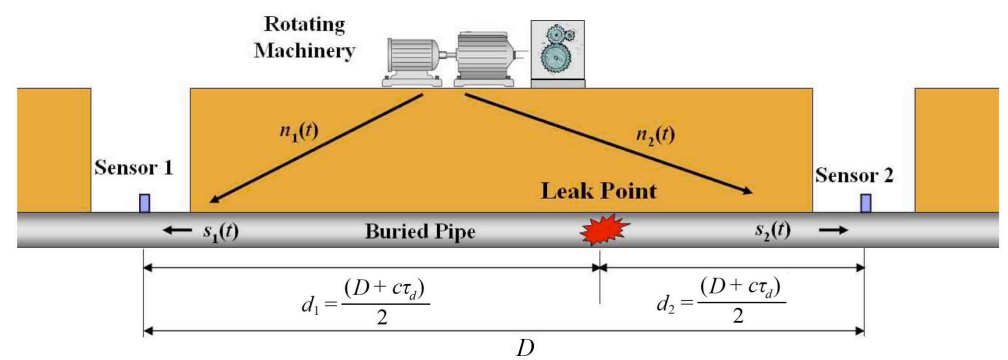

Fig. 3. Schematic Diagram of Typical Measurement Arrangement for Leak Detection of a Buried Pipe in a Power Plant 
The cross-correlation function of the signals $x_{1}(t)$ and $x_{2}(t)$ is obtained by [12]

$$
C_{12}(\tau)=E\left[x_{1}(t) x_{2}(t+\tau)\right]
$$

where $\tau$ is the lag of time, and $E[\cdot]$ is the expectation operator.

If the background noise due to rotating machinery at each sensor is assumed to be uncorrelated with the leakage signals, then the cross-correlation function of the signals $x_{1}(t)$ and $x_{2}(t)$ can be expressed as

$$
C_{12}(\tau)=E\left[s_{1}(t) s_{2}(t+\tau)\right]+E\left[n_{1}(t) n_{2}(t+\tau)\right]
$$

The first term in Eq. (4) represents a cross-correlation due to leakage signals, and the second term represents a cross-correlation due to machinery noise.

If a leak exists and $n_{1}(t)=0$ and $n_{2}(t)=0$, a single peak can be found in the cross-correlation function, as shown in Fig. 1. The location of the leak can be calculated by using the relations between the time $\operatorname{delay}\left(\tau_{d}\right)$, the distance between sensors $(D)$, and the wave propagation speed $(c)$ [13 15] of a buried pipe as expressed by Eqs. (5) and (6).

$$
\begin{gathered}
d_{1}-d_{2}=\tau_{d} \cdot c \\
d_{1}=\left(D+c \tau_{d}\right) / 2, \quad d_{2}=\left(D-c \tau_{d}\right) / 2
\end{gathered}
$$

However, in practical situations, the machinery noise component (the second term of Eq. (4)) introduces many peaks in the cross-correlation function in addition to the peak corresponding to the true time $\operatorname{delay}\left(\tau_{d}\right)$ resulting from a propagating leak signal. These additional peaks due to rotating machinery noise make it difficult to find out the true time delay of the leakage signals. The effect of the machinery on the performance of the cross-correlation technique will be demonstrated by performing a simulation in section 3.1 .

Therefore, in order to enhance the leak detection capability of the cross-correlation method, it is necessary to effectively remove the mechanical noise component $\left(2^{\text {nd }}\right.$ term in Eq. (4)).
It is well known that the leak signal can be modeled as a random signal[10,16] as shown in Fig. 4 (a). On the other hand, the vibration signal of rotating machinery can be modeled as a superposition of periodic signals. In the time domain, it is not easy to distinguish the leak signals $\left(s_{1}(t)\right.$ and $\left.s_{2}(t)\right)$ from the noise signals $\left(n_{1}(t)\right.$ and $\left.n_{2}(t)\right)$. But, in the frequency domain, the leak signal may be expressed as a flat broadband spectrum, and the vibration signal of the rotating machinery can be expressed as a harmonic peak component as shown in Fig. 4 (a) and (b). Therefore, in the frequency domain, it is possible to distinguish the leak signal components from the noise signal components.

In this work, based on this frequency characteristic of the leak signal and rotating machinery noise, a modified cross-correlation technique that can effectively remove the rotating machinery noise component is suggested.

Fig. 5 illustrates the method for reducing the machinery noise component in the frequency domain. The noise

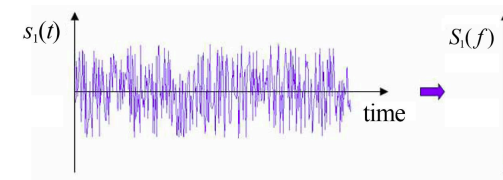

(a)
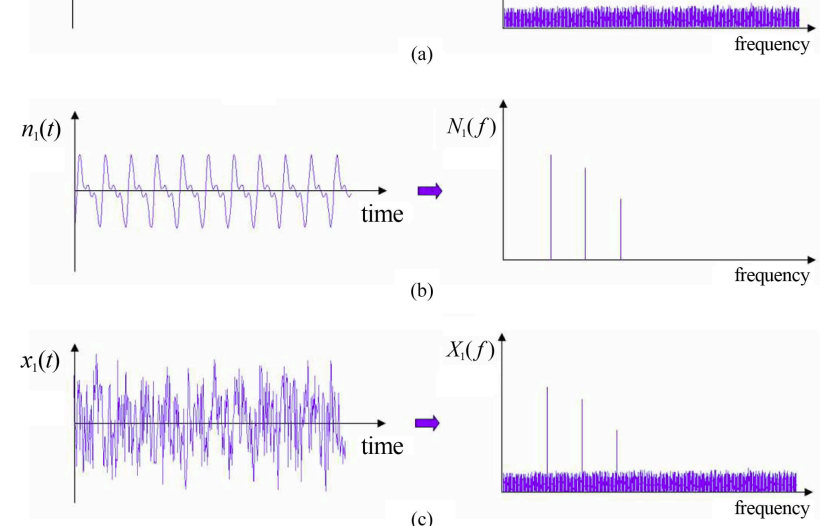

Fig. 4. Frequency Characteristics of the Random(Leakage) Signal and Periodic(Rotating Machinery) Signal, (a) Random Signal, (b) Periodic Signal, (c) Random Signal + Periodic Signal

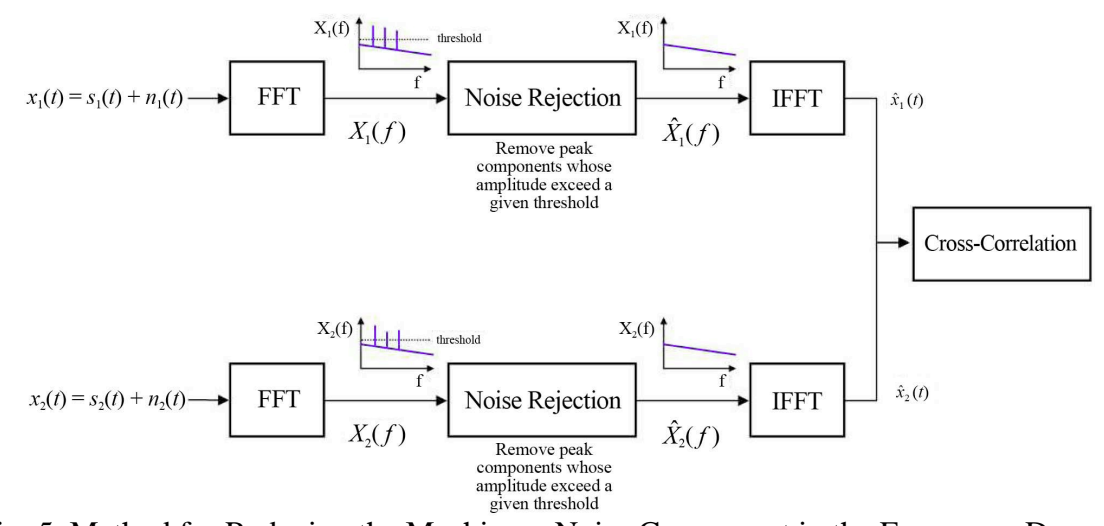

Fig. 5. Method for Reducing the Machinery Noise Component in the Frequency Domain 
rejection algorithm of the proposed method consists of three steps as follows.

i) Step 1: FFT of the measured signals $\left(x_{1}(t)\right.$ and $\left.x_{2}(t)\right)$

In order to distinguish the leak signal components from the noise signal components, one can perform FFT(Fast Fourier Transform) of the vibration signals measured at sensor locations 1 and 2. Then, in the frequency domain, the measured vibration signals can be expressed as

$$
\begin{array}{r}
X_{1}(f)=S_{1}(f)+N_{1}(f) \\
X_{2}(f)=S_{2}(f)+N_{2}(f)
\end{array}
$$

where $S_{1}(f)$ and $S_{2}(f)$ represent the frequency spectrum of leakage signals measured at sensor locations 1 and 2, respectively. $N_{1}(f)$ and $N_{2}(f)$ represent the frequency spectrum of machinery noise signals measured at sensor locations 1 and 2, respectively.

ii) Step 2: Machinery noise rejection in the frequency domain

Since the periodic noise components due to rotating machinery are represented as peaks in the frequency domain, in order to remove the peak components, peak rejection criteria that remove peak components whose amplitude exceed a given threshold can be used as shown in Fig. 5.

The threshold level $\left(L_{t h}\right)$ should be larger than the ampli- tude of the leakage signal in the frequency domain $(\bar{s})$, as shown in Fig. 6 (a) and (c). The lower bound of the threshold level $\left(L_{t h, l o w e r}\right)$ can be roughly estimated by using the auto-correlation function $\left(R_{x x}(\tau)\right)$ of the measured signal as shown in Fig. 6 (c). Then, the threshold level can be chosen by a user based on the lower and upper bounds of $L_{t h}($ Eq. (9)).

$$
\begin{gathered}
L_{\text {th,lower }} \cong\left(\left[R_{x x}(0)-R_{x x}(\tau)_{\tau \neq 0, \max }\right] / B_{f}\right)^{1 / 2} \\
L_{t h, \text { lower }}<L_{t h}<\text { amplitude of the maximum peak }
\end{gathered}
$$

Practically, the threshold level $\left(L_{t h}\right)$ can be determined based on the observation of the experiment data $\left(X_{1}(f)\right.$, $\left.X_{2}(f)\right)$ on a case by case basis. In this work, the threshold level is set as $10 \%$ of the amplitude of the maximum peak ( $L_{\text {th, upper }}$ ).

As an alternative method to reject machinery noise, it is possible to use notch filters or zero-value replacements at a narrow frequency band for eliminating harmonic noise in the frequency domain.

iii) Step 3: IFFT of $\hat{X}_{1}(f)$ and $\hat{X}_{2}(f)$ and calculation of the cross-correlation function

After removing the tonal noise components $\left(N_{1}(f)\right.$ and $\left.N_{2}(f)\right)$, the noiseless spectrum $\left(\hat{X}_{1}(f)\right.$ and $\left.\hat{X}_{2}(f)\right)$ can be transformed into the time domain $\left(\hat{x}_{1}(t), \hat{x}_{2}(t)\right)$ by using
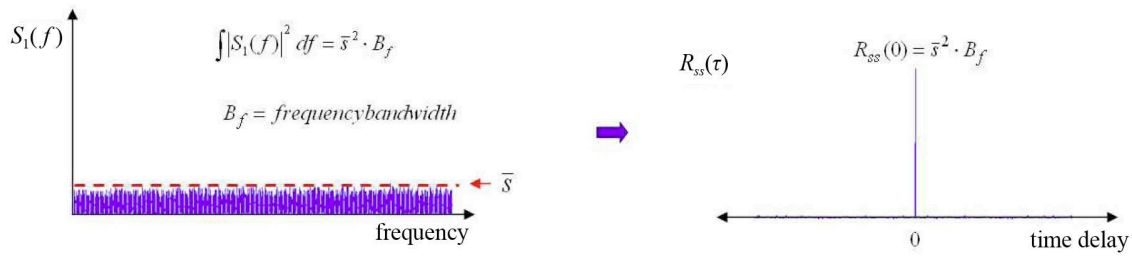

(a)
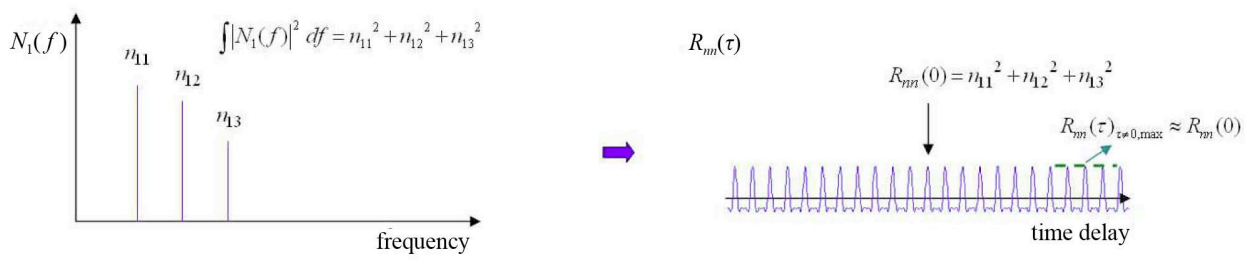

(b)
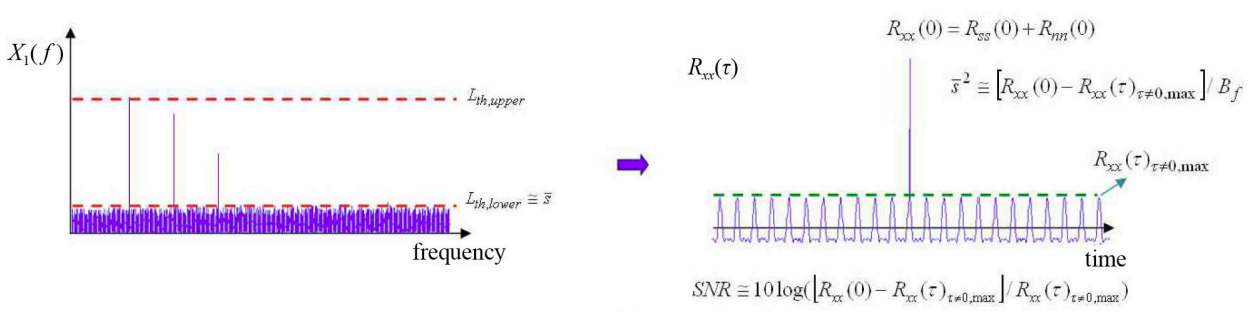

(c)

Fig. 6. Relation between the Auto-correlation Function and the Frequency Spectrum of the Random/ Periodic Signal, (a) Random Signal, (b) Periodic Signal, (c) Random Signal + Periodic Signal 
IFFT(Inverse Fast Fourier Transform) as shown in Fig. 5. Then, the cross-correlation function of the noiseless leak signals can be obtained as

$$
\hat{C}_{12}(\tau)=E\left[\hat{x}_{1}(t) \hat{x}_{2}(t+\tau)\right]
$$

By removing the tonal peaks due to rotating machinery as shown in Fig. 5, reliability and precision of the crosscorrelation method can be enhanced. In section 3.2, the feasibility of the proposed method will be demonstrated by experiments.

\section{SIMULATIONS AND EXPERIMENTS}

\subsection{Simulations}

The effect of the rotating machinery noise on the crosscorrelation technique is investigated by performing numerical simulations. For this purpose, a zero mean Gaussian random noise is modeled as a leakage signal. The mechanical noise due to rotating machinery with BPF(blade passing frequency) of $240 \mathrm{~Hz}$ and its harmonic components are artificially generated and mixed with the random(leakage) signals as shown in Fig. 7.

Fig. 8 represents the simulation results of the crosscorrelation technique when the levels of mechanical noise increase. The SNR(signal-to-noise ratio) is defined as the power ratio of leak signal to mechanical noise. By observing Fig. 8 (a), one can see the peak corresponding to the

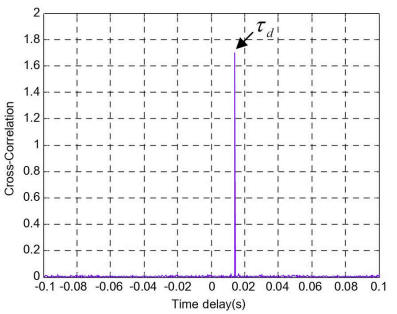

(a)

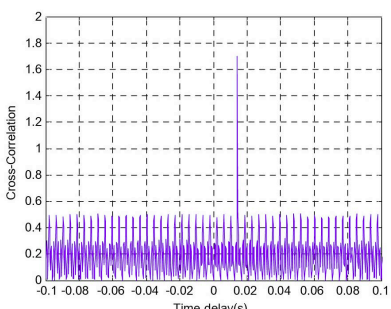

(c)

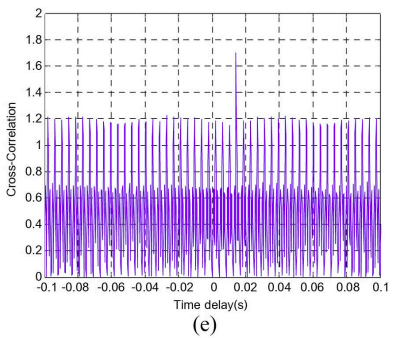

Fig. 8. Effects of Machinery Noise on the Cross-correlation Technique, (a)Without Noise, (b) With Noise(SNR=10dB), (c) With Noise $(\mathrm{SNR}=6 \mathrm{~dB})$, (d) With Noise $(\mathrm{SNR}=4 \mathrm{~dB})$ (e) With Noise $(\mathrm{SNR}=3 \mathrm{~dB})$, (f) With Noise $(\mathrm{SNR}=0 \mathrm{~dB})$
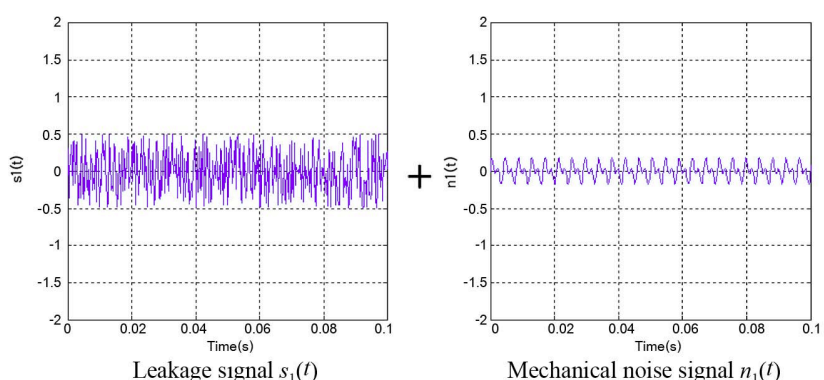

(a)
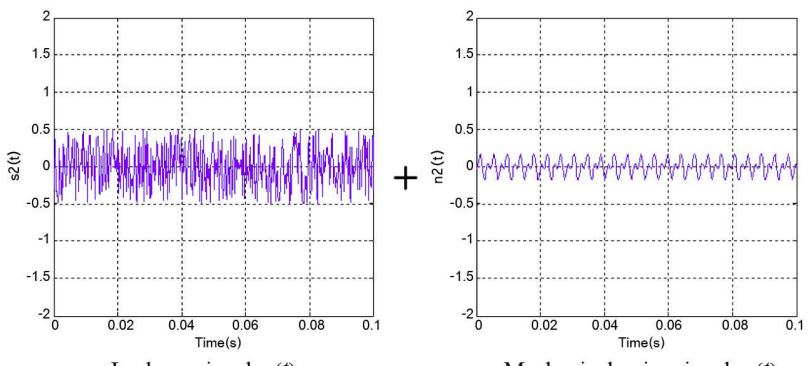

Mechanical noise signal $n_{2}(t)$

(b)
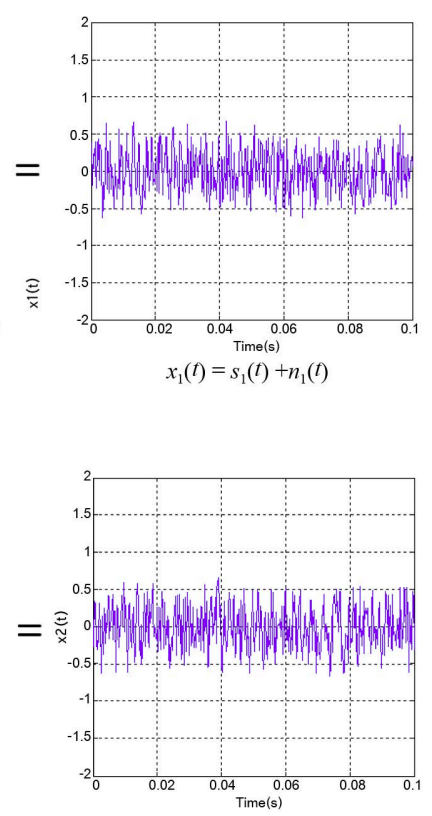

$x_{2}(t)=s_{2}(t)+n_{2}(t)$

Fig. 7. Leakage Signal and Machinery Noise used in the Numerical Simulation, (a) Signal at Point $1\left(x_{1}(t)\right)$, (b) Signal at point $2\left(x_{2}(t)\right)$ 

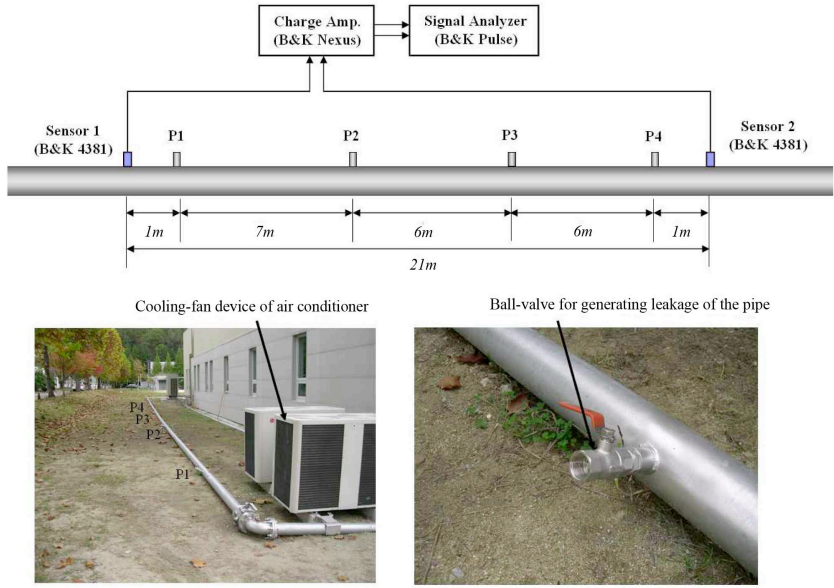

Fig. 9. Experimental Setup and Schematic of the Data Acquisition of the Leakage Signal

true time delay $\left(\tau_{d}\right)$ of the propagating leakage signal. However, by observing Fig. 8 (c), (d), (e), and (f), as the SNR decreases, one can see that it is impossible to find the peak component corresponding to the true time delay due to the leakage signal. Therefore, one can confirm that the presence of the rotating machinery noise is highly undesirable for estimating the true time $\operatorname{delay}\left(\tau_{d}\right)$ of the leakage signal, and the conventional cross-correlation technique could be problematic for locating leaks.

\subsection{Experiments}

An experiment has been performed to investigate the validity of the proposed technique for leakage detection of a pipe. Fig. 9 represents the experimental setup and a schematic of the data acquisition. To simulate a noisy environment, the pipe for the leakage test was installed near an air conditioner cooling fan as shown in Fig. 9. Two sensors(B\&K 4381) were used for measuring acceleration signals at either side of the test pipe as shown in Fig. 9. The leakage signals of the pipe were artificially generated by opening the ball-valves located at four points (P1 P4) along the test pipe.

Fig. 10 (a) and (b) represent measured acceleration signals and the cross-correlation function due to leakage at point P1. In Fig. 10 (b), one can see that it is not easy to find the peak component corresponding to the true time delay resulting from the propagating leak signal. Fig. 10 (c) represents the frequency $\operatorname{spectrum}\left(X_{1}(f)\right.$ and $\left.X_{2}(f)\right)$ of the measured vibration signal. The SNR of the measured signals $X_{1}(f)$ and $X_{2}(f)$ are estimated as $5.5 \mathrm{~dB}$ and $3.9 \mathrm{~dB}$, respectively. The SNR is estimated by using the information of the auto-correlation of the measured signal as shown in Fig. 6 (c). In order to reduce the tonal noise components, the noise rejection algorithm(shown in Fig 5) is applied for $X_{1}(f)$ and $X_{2}(f)$. The threshold level for tonal noise rejection is chosen as $10 \%$ of the amplitude of the maximum peak $\left(L_{t h}=0.1 L_{t h \text {, upper }}\right)$. Fig. $10(\mathrm{~d})$ represents

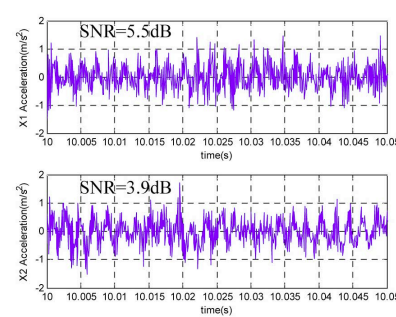

(a)
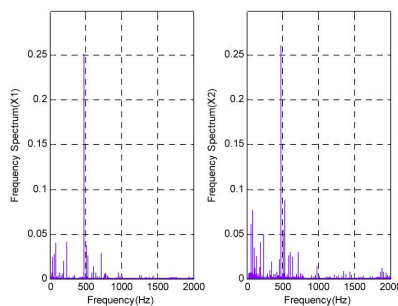

(c)
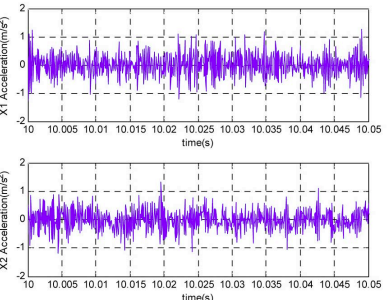

(e)

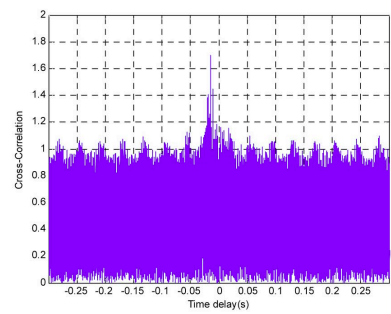

(b)

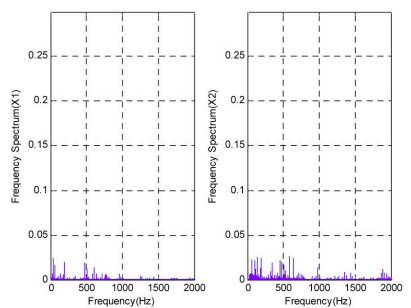

(d)

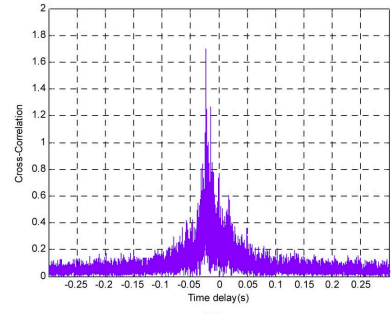

(f)
Fig. 10. Result for the Leak Detection Experiment (Leakage at Point P1), (a) Measured Acceleration Signals at Both Sides of the Pipe(Sensors 1 and 2), (b) Cross-correlation Function before

Noise Rejection, (c) Frequency Spectrum before Noise

Rejection, (d) Frequency Spectrum after Noise Rejection, (e) Time Signal after Noise Rejection, (f) Cross-correlation Function after Noise Rejection

the frequency spectrum $\left(\hat{X}_{1}(f)\right.$ and $\left.\hat{X}_{2}(f)\right)$ after removing the periodic noise components. Fig. 10 (e) represents the time signals $\left(\hat{x}_{1}(t)\right.$ and $\left.\hat{x}_{2}(t)\right)$ after removing the noise components. Fig. 10 (f) demonstrates the noise rejection capability of the proposed method. By comparing Fig. 10 (f) with Fig. 10 (b), one can observe that the machinery noise component has been reduced remarkably and it is possible to identify the time delay of the leakage signal by applying the noise rejection technique(Fig. 5) to the noisy signal.

Fig. 11 (b) and (c) represent the cross-correlation function and frequency spectrum of the measured signals due to leakage at point P2. Similar to the case of Fig. 10, one can see that the additional noise components due to the rotating machinery can be effectively removed from the cross-correlation function by applying the proposed noise rejection technique to the noisy leakage signal.

Figs. 12 and 13 represent results for leak detection experiments when the leakage occurs at points $\mathrm{P} 3$ and $\mathrm{P} 4$, respectively. These experimental results also demonstrate that even in a noisy environment, the proposed method can effectively remove the tonal noise components, and one 
(a)

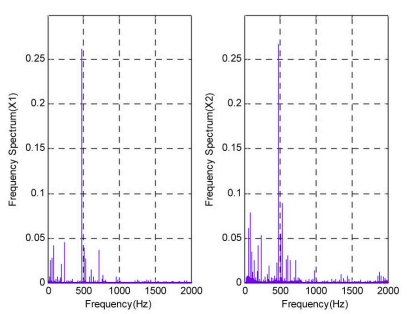

(c)

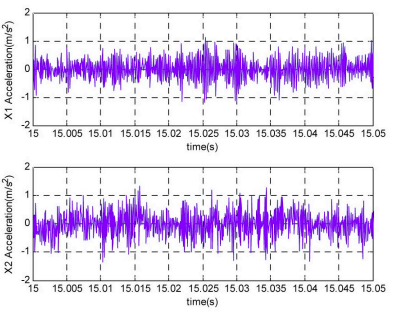

(e)

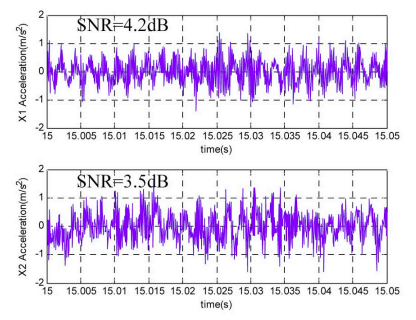

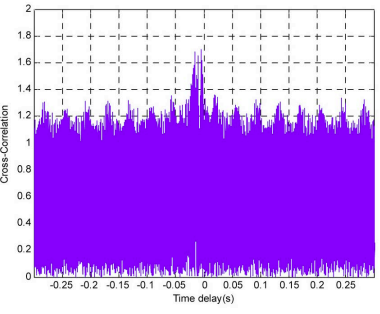

(b)

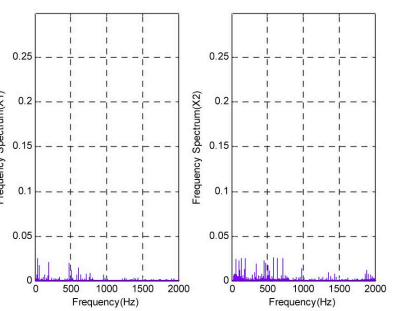

(d)

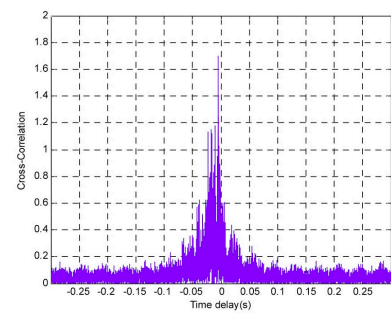

(f)
Fig. 11. Result for the Leak Detection Experiment (Leakage at Point P2), (a) Measured Acceleration Signals at Both Sides of the Pipe(Sensors 1 and 2), (b) Cross-correlation Function before Noise Rejection, (c) Frequency Spectrum before Noise Rejection, (d) Frequency Spectrum after Noise Rejection, (e)

Time Signal after Noise Rejection, (f) Cross-correlation Function after Noise Rejection

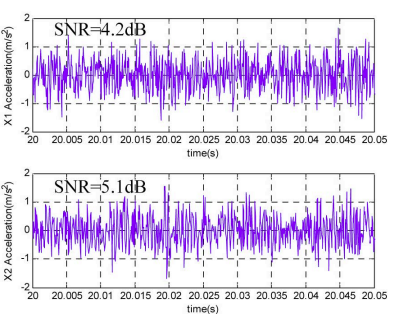

(a)

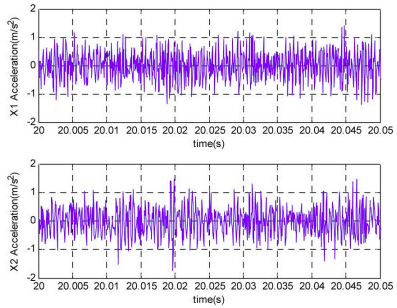

(c)

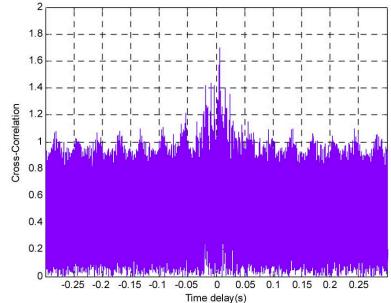

(b)

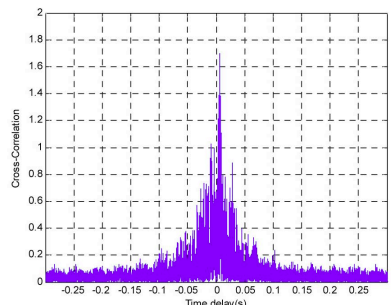

(d)
Fig. 12. Result for the Leak Detection Experiment (Leakage at Point P3), (a) Measured Acceleration Signals at Both Sides of the Pipe(Sensors 1 and 2), (b) Cross-correlation Function before Noise Rejection, (c) Time Signal after Noise Rejection,

(d) Cross-correlation Function after Noise Rejection

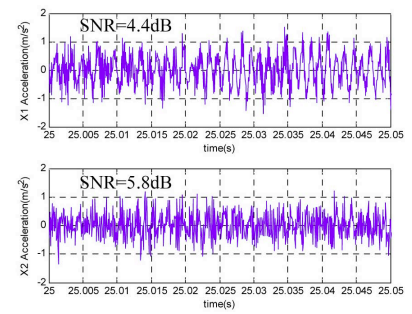

(a)
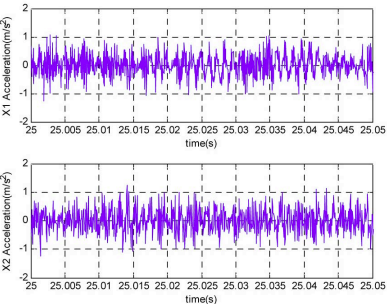

(c)

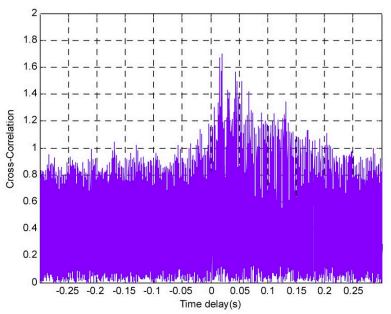

(b)

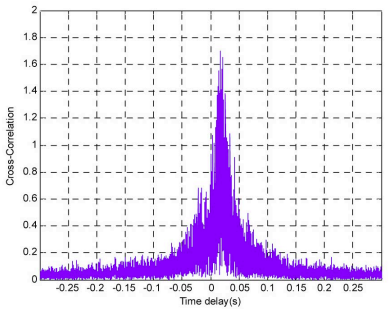

(d)
Fig. 13. Result for the Leak Detection Experiment (Leakage at Point P4), (a) Measured Acceleration Signals at Both Sides of the Pipe(Sensors 1 and 2), (b) Cross-correlation Function before

Noise Rejection, (c) Time Signal after Noise Rejection, (d) Cross-correlation Function after Noise Rejection

can find the time delay due to leakage of the pipe.

From above, it has been found that the proposed method provides a useful means for estimating the time delay of the leakage signal in a noisy environment throughout Figs. 10 (f), 11 (f), 12 (d), and 13 (d). Therefore, it is expected that the reliability of leakage point estimation could be enhanced when the proposed technique is applied to a leakage estimation problem for the buried pipes of a power plant.

\section{CONCLUSION}

The objective of this work is to enhance the leak point estimation capability of the cross-correlation technique in a tonal noisy environment. For this purpose, a modified cross-correlation method that can effectively remove the rotating machinery noise component was proposed. Also, experiments were carried out to verify the validity of the proposed method. The experimental results demonstrate that the performance of the cross-correlation method can be enhanced by reducing the periodic noise components due to mechanical equipment. It is expected that the proposed method can be utilized for leak detection of buried pipes in a tonal noisy environment.

\section{REFERENCES}

[1] R. Iserman, "Process Fault Detection on Modeling and Estimation Methods - A Survey," Automatica, Vol. 20(4), pp. 387-404, (1984).

[2] Y.-H. Cho, "A Study of Guided Waves for Buried Pipe 
Inspection," Proceeding of the 2010 KPVP, Korea, pp. 3334, (2010).

[ 3 ] G. J. Weil, "Non Contact, Remote Sensing of Buried Water Pipeline Leaks Using Infrared Thermography," Water Resources Planning and Management and Urban Water Resources, pp. 404-407, (1993).

[4] L. Billman et al., "Leak Detection Methods for Pipelines," Proceeding of the $9^{\text {th }}$ IFAC Congress, Hungary, (1984).

[5] R. Iserman, Fault-Diagnosis Applications: Model-based Condition Monitoring, Chapter 7, Springer, 2011.

[6] H. V. Fuchs, "Ten Years of Experience with Leak Detection by Acoustic Signal Analysis," Applied Acoustics, Vol. 33, pp. 1-19, (1991).

[ 7 ] D. A. Liston et al., "Leak Detection Techniques," Journal of New England Water Works Association, Vol. 1206(2), pp.103-108, (1992).

[ 8 ] Y. Gao, M. J. Brennan, P. F. Joseph, "A Comparison of Time Delay Estimators for the Detection of Leak Noise Signals in Plastic Water Distribution Pipes," Journal of Sound and Vibration, Vol. 292, pp. 552-570, (2006).

[9] Y.-S. Lee and D.-J. Yoon, "An Algorithm for Leak Point Detection of Underground Pipelines," Proceedings of the
KSNT Annual Spring Conference, pp. 212-218, (2004).

[10] Y.-S. Lee, "Leak Point Detection of Underground Water Pipelines," Journal of the Korean Society for Noise and Vibration Engineering, Vol. 17(1), pp. 16-21, (2007).

[11] K. Kazuhisa, "Method and Apparatus for Detecting Leakage Position in Piping," Japan Patent No. JP-2004-125628A, 2004.

[12] J. S. Bendat and A. G. Piersol, Random Data, Wiley, 1986.

[13] R. J. Pinnington and A. R. Briscoe, "Experimentally Applied Sensor for Axisymmetric Waves in a Fluid Filled Pipe," Journal of Sound and Vibration, Vol. 173(4), pp. 503-516, (1994).

[14] J. M. Muggleton et al., "Wavenumber Prediction of Waves in Buried Pipes for Water Leak Detection," Journal of Sound and Vibration, Vol. 249(5), pp. 939-954, (2002).

[15] J. M. Muggleton et al., "Axisymmetric Wave Propagation in Fluid-Filled Pipes: Wavenumber Measurements in in Vacuo and Buried Pipes," Journal of Sound and Vibration, Vol. 270, pp. 171-190, (2004).

[16] Osama Hunaidi, Wing T. Chu, "Acoustical Characteristics of Leak Signals in Plastic Water Distribution Pipes," Applied Acoustics, Vol. 58, pp. 235-254, (1999). 